\title{
PDB-Tools Web: A user-friendly interface for the manipulation of PDB files
}

\author{
Brian Jimenez-Garcia ${ }^{1}$, João Teixeira ${ }^{2}$, Mikael Trellet $^{1}$, João Rodrigues ${ }^{3}$, and Alexandre \\ M.J.J. Bonvin ${ }^{1}$
}

${ }^{1}$ Utrecht University Faculty of Science

${ }^{2}$ The Hospital for Sick Children

${ }^{3}$ Stanford University School of Medicine

August 5, 2020

\begin{abstract}
The Protein Data Bank (PDB) file format remains a popular format used and supported by many software to represent coordinates of macromolecular structures. It however suffers from drawbacks such as error-prone manual editing. Because of that, various software toolkits have been developed to facilitate its editing and manipulation, but, to date, there is no online tool available for this purpose. Here we present PDB-Tools Web, a flexible online service for manipulating PDB files. It offers a rich and user-friendly graphical user interface that allows users to mix-and-match more than 40 individual tools from the pdb-tools suite. Those can be combined in a few clicks to perform complex pipelines, which can be saved and uploaded. The resulting processed PDB files can be visualized online and downloaded. The web server is freely available at https://wenmr.science.uu.nl/pdbtools.
\end{abstract}

\section{Hosted file}

PDB-Tools-Web_proteins.pdf available at https://authorea.com/users/348899/articles/474092pdb-tools-web-a-user-friendly-interface-for-the-manipulation-of-pdb-files 